\title{
Nutrition in the intensive care unit
}

\author{
Chase C. Hansen MBA, Sharmila Dissanaike MD
}

\begin{abstract}
Nutrition has evolved into an integral part of modern critical care for both medical and surgical patients. Initially a neglected field that was considered peripheral to the medical care of the patient, it is now recognized as a valid specialty with a significant body of evidence to support current practice. Multiple international societies now issue evidence-based guidelines to help clinician optimize their patients' nutrition. However, adherence to these guidelines throughout the country is poor, and education in nutrition is still lagging in medical schools. As a result, many intensive care units fail to meet benchmarks for adequate nutrition. This review article examines briefly the evolution of nutrition as a part of ICU care, nutrition education for physicians, the evolution of evidence-based guidelines, and barriers to their implementation. The authors also highlight four key areas where evidence-based recommendations exist - timing of initiation of enteral feeding, calculating and meeting total daily requirements, use of gastric residual volumes to guide delivery, and interruptions of feeding for procedures - but are currently often not followed. Reasons for this lack of adherence are explored, and potential solutions discussed.
\end{abstract}

Key words: Nutrition, intensive care unit (ICU), malnutrition, critically ill, guidelines

\section{INTRODUCTION}

The evolution of modern nutrition therapy (NT) began in the mid 1940's as physicians managed a large number of wounded soldiers and civilians. Over time, they noticed a correlation between patient outcomes and perioperative nutrition. In the 1950's and 60's, clinicians advanced their investigation of NT's role in critically ill patients by varying the nil per os (NPO) status of patients while attempting to optimize patient outcomes. ${ }^{1}$ In 1986, a trauma group at the University of Washington in Seattle published a randomized clinical trial comparing enteral nutrition (EN) with parenteral nutrition (PN), confirming the importance of EN as an integral component of medical management. ${ }^{2}$

Corresponding author: Sharmila Dissanaike MD Contact Information: Sharmila.Dissanaike@ttuhsc.edu DOI: $10.12746 /$ swrccc 2015.0310.127
In the past decade, researchers and clinicians have increased our knowledge of optimizing the nutritional management in patients. These research driven attempts to understand how nutrition improves wound healing and recovery from critical illness have led to the current publication of American, Australian, Canadian, and European guidelines for NT (see Table 1). ${ }^{3-7}$ These guidelines are needed since nearly $40 \%$ of patients admitted to the hospital are malnourished at admission and over $2 / 3$ of hospitalized patients experience deteriorating nutritional status while under "proper" hospital care..$^{8,9}$ Despite increased awareness of the importance of nutrition for recovery from critical illness, there remains a large gap between recommendations and application. Studies in the United States report that the percentage of critically ill patients attaining NT goals within the first 72 hours varies from $30 \%$ to $85 \% .{ }^{3}$ One of the reasons behind this deficit may be a lack of education in healthcare providers. Most medical train- 
ing programs in the US have limited exposure to nutrition education, and studies have shown a notable discordance between nutrition knowledge and application among physicians, fellows, and residents. ${ }^{8,10-13}$ This disunity in nutrition management in medical professionals is worrisome and identifies an opportunity to improve outcomes through education.

\section{GUIDELINES}

In 1926 the first intensive care unit (ICU) was developed at Johns Hopkins University. Initially, the role of NT was acknowledged as a minor adjunctive therapy. The evolution of NT over the past century has led to a sophisticated, proactive therapy that definitely affects patient outcomes, and national guidelines have continually evolved. While the value of these guidelines is rarely refuted, many healthcare providers either trust their own experience-based knowledge or do not have the means needed to comply with them. ${ }^{14}$ Thus, these obstacles reduce the practical application of guidelines internationally.

Barriers to NT implementation have been studied several ways. One method of addressing this issue is the utilization of standardized protocols. ${ }^{15}$ This approach has revolutionized medical outcomes in other fields, and its influence is further supported by studies suggesting that protocols for NT improve compliance with the established guidelines and optimize patient outcomes. Education programs are being thoroughly investigated to promote provider confidence with the literature and national guidelines. Preliminary studies are positive and show increased short- and long-term compliance with the guidelines after minimal, but consistent, training programs. ${ }^{8,10,11}$

\section{Educational competence of heAlthcare}

\section{PROFESSIONALS}

Physicians at Rush University Medical Center were surveyed to ascertain their understanding and application of NT. ${ }^{8}$ All groups, attending physicians, fellows, and residents, agreed and placed high value (4.5 out of 5 point scale) on the significance of nutrition in the outcomes of critically ill patients. Next they were questioned on their comfort level with the NT of- fered at their institution. Again, all three groups voiced similar responses but rated their comfort as 3.5 out of 5 . The study also surveyed their knowledge about the initiation of enteral feeding (EF). The group's responses ranged from waiting 1.8 days of NPO status to 2.6 days, with the overwhelming majority choosing EN over PN. While these scores are not significantly different from one another, the amount of variation increases with the complexity of management questions. When asked about more complex management, clearly addressed by national guidelines, the percentage of responses held near an even 50:50. In these situations, patient management is essentially determined by the flip of a coin. Understanding the effects of post-pyloric feeding tube placement or whether an ileus is an absolute contraindication is straightforward management question, which ought to be stressed during ICU training, yet they still returned a 50:50 response.

This study does not report unique findings. Darawad, et al. and others also reported that healthcare professionals commonly understand the significance of nutrition in managing patients, but their knowledge is inadequate. ${ }^{13,16,17}$ Even in hospitals with competent staff and trained professionals, nutritional support was sporadic and hampered by inconsistency and miscommunication.

Current NT education is limited throughout the United States. Indeed, healthcare professional training programs sparsely address the basic science and clinical skills associated with NT. ${ }^{16}$ For example, US-based medical schools are required to provide only 10 hours of lecture devoted to NT education. Even though the American Society for Parenteral and Enteral Nutrition (ASPEN) recommends spending 25 hours covering NT, only $33 \%$ of medical schools actually attain this objective..$^{11,16}$ One study recently demonstrated optimal implementation of NT education as an integrated course, one in which students learn the management of drugs, surgery, and fluids and electrolytes associated with NT. This model of education increased both short- and long-term memory of the national guidelines and produced more unified and consistent management. ${ }^{16}$ Programs should develop a system that incorporates residents, fellows, 
and attending physicians.

In an attempt to mitigate this discordance and improve provider confidence in NT, we analyzed recently published studies to determine the issues that were commonly reported. ${ }^{8-12}$ The results indicate that delayed initiation of EN, determination of total caloric need, utility of residual volumes, interruptions of tube feeding, and prolonged fasting periods were the is- sues with highest discordance rates. We will briefly address each of these.

\section{Delayed initiation of EN}

The national guidelines for initiation of EN are similar across the board and state that EN should be initiated within the first 24-48 hours following admission (see Table 1). However compliance with

\section{Table 1 Key Recommendations from A.S.P.E.N. and E.S.P.E.N. Guidelines ${ }^{3,4,6,7,12}$}

\section{Initiation of Enteral Nutrition}

EN is the preferred route of feeding over PN for the critically ill patient who requires nutrition support therapy. (Grade B) EN should be established within the first $24-48$ hours following admission. (Grade C)

In the ICU patient population, neither the presence nor absence of bowel sounds nor evidence of passage of flatus and stool is required for the initiation of enteral feeding. (Grade B)

Either gastric or small bowel feeding is acceptable in the ICU setting. Critically ill patients should be fed via an enteral access tube placed in the small bowel if at high risk for aspiration or after showing intolerance to gastric feeding. (Grade C)

Use of Parenteral Nutrition

If early EN is not feasible or available the first 7 days following admission to the ICU, no nutrition support therapy should be provided. (Grade C)

Avoid PN in patients who tolerate EN and can be fed approximately to the target values. (Grade A)

PN therapy provided for a duration of $<5-7$ days would be expected to have no outcome effect and may result in increased risk to the patient. Thus, PN should be initiated only if the duration of therapy is anticipated to be $\geq 7$ days. (Grade: B)

No difference in 30-day mortality associated with either EN or PN for early NT

All patients who are not expected to be on normal nutrition within 3 days should receive PN within 24 to $48 \mathrm{~h}$ if EN is contraindicated or if they cannot tolerate EN. (Grade: C)

All patients receiving less than their targeted enteral feeding after 2 days should be considered for supplementary PN.

(Grade: C)

\section{Dosing of Enteral Nutrition}

The target goal of EN (defined by energy requirements) should be determined and clearly identified at the time of initiation of nutrition support therapy. (Grade: C)

Efforts to provide $>50 \%-65 \%$ of goal calories should be made in order to achieve the clinical benefit of EN over the first week of hospitalization. (Grade: C)

Initiating supplemental PN prior to a 7-10 day period in the patient already receiving EN does not improve outcome and may be detrimental to the patient. (Grade: $\mathrm{C}$ )

\section{Monitoring Tolerance and Adequacy of Enteral Nutrition}

Holding EN for gastric residual volumes $<500 \mathrm{~mL}$ in the absence of other signs of intolerance should be avoided. (Grade:B) The time period that a patient is made nil per os (NPO) prior to, during, and immediately following the time of diagnostic tests or procedures should be minimized to prevent inadequate delivery of nutrients and prolonged periods of ileus. Ileus may be propagated by NPO status. (Grade: C)

Use of enteral feeding protocols increases the overall percentage of goal calories provided and should be implemented.

(Grade: C)

In all intubated ICU patients receiving EN, the head of the bed should be elevated $30^{\circ}-45^{\circ}$. (Grade: C)

Abbreviations: $\mathrm{EN}=$ Enteral Nutrition; $\mathrm{PN}=$ Parenteral Nutrition

Grading: A supported by $\geq 2$ level I investigations; B supported by 1 level I investigation; C supported by level II investigations only 
with this guideline is poor, with multiple reasons often given as justification for delaying the start of EN, including uncertainty of whether EN will be required or impending extubation in an intubated patient. Several studies of NT education programs implemented in training hospitals reported higher rates of early EN initiation (pre-educational program $24 \%$ vs. post-educational program 60\%) and more adequate EN management as a percentage of goal (pre-EP $74 \%$ vs. post-EP 96\%) after completion of the NT course. ${ }^{10,15}$ The outcomes of compliance with this single guideline have not been studied, but when incorporated with the observance of multiple guidelines, patient outcomes improved. ${ }^{18}$ The standard of early initiation is becoming a marker for the quality of care in patients in the critical care units. ${ }^{15}$ The delayed initiation of EN is not justified in the setting of modern evidence-based medicine.

\section{Total caloric needs and management}

Current medical practices encourage that a target goal of EN (defined by energy requirements) should be determined and clearly identified at the time of initiation of nutrition support therapy (see Table 1). In spite of this guideline, none of the national guidelines recommend a specific, "gold standard" tool to calculate the total caloric requirements of a patient. This complex calculation is based on the severity of the patient's medical conditions, the patient's body mass index, and the clinician's experience and plan. One tool often utilized in this calculation is indirect calorimetry. ${ }^{13,15}$ Even with this tool, it is challenging to determine the exact caloric needs of each patient.

Adding to the complexity of the situation is the fact that standard markers of nutrition are often unreliable in critical illness. The inflammatory response common to trauma, sepsis, and many forms of acute critical illness stimulates the production of positive acute phase reactants, such as C-reactive protein, while inhibiting production of albumin, retinol binding protein, and pre-albumin (transthyretin) which are the most commonly used nutritional markers. This inflammation-induced decrease in production limits their utility as markers in critical illness and likely reflects the course of the primary illness rather than the nutritional status. ${ }^{3,19}$ A review article on this subject affirms the weak correlation of most acute phase reactants and clinical outcomes in the ICU; however, specific proteins measured serially, namely C-reactive protein, transthyretin, and retinol-binding protein, appear to be more sensitive to the nutritional status in those patients. ${ }^{19}$

\section{Gastric residual volumes}

Another common problem is ensuring that the total prescribed calories for a critically ill patient are actually being delivered. Historically patients have often had their enteral feeding reduced or withheld based on gastric residual volumes (GRVs) which were used as a marker of food intolerance; this resulted in patients receiving significantly fewer calories than intended. The use of GRVs a potential factor preventing successful management since some providers errantly use GRV as a clinical tool to correlate gastric emptying and abdominal distension. Other clinicians inaccurately use GRV as a proxy for risk of pneumonia, regurgitation, or aspiration. While there is literature evidence to support both sides of the argument, the majority favors the idea that GRV is not an appropriate tool to assess the previously mentioned entities. ${ }^{3,8,20-22}$ In fact, a recent French study claimed that the omission of GRV monitoring might be beneficial and improve patient outcomes. ${ }^{22}$ The lack of GRV data in this study did not influence rates of ICU-related infection, ICU length of stay (LOS), or mortality when compared to their control arm. One strong argument against the use of GRV is that it leads clinicians to premature cessation and inappropriate withholding of NT, essentially starving critically ill patients. ${ }^{20}$ ASPEN, the Society of Critical Care Medicine (SCCM), and other national societies recommend the avoidance of holding EN for a gastric residual volume $<500$ $\mathrm{mL}$ in the absence of other signs of intolerance (see Table 1). There are other clinical tools to help providers in their assessment of patient tolerance to EF. The time-intensive and costly nature of scintigraphy limits its use in the ICU setting; newer techniques, such 
as refractometry and gastric impedance monitoring, show some promise. ${ }^{21}$

To further address the issue of poorly managed NT, national guidelines additionally recommend the use of EF protocols, and claim that its use alone increases the overall percentage of goal calories provided to critically ill patients (see Table 1). Such protocols provide specific orders that determine the optimal sequence of NT initiation, goal rates, frequency of flushing, and other easily overlooked precautions. Compliance with NT protocols minimizes variation in medical management, increases the percentage of nutrition actually delivered, and effectively reduces patient intolerance and GRV. ${ }^{23}$ While utilizing protocols takes time for development and implementation, and may reduce provider autonomy, the benefits probably outweigh the potential losses.

\section{Prolonged fasting and interruptions of feeding for procedures}

Critically ill patients, especially those with primarily surgical problems, are frequently made NPO for operations and procedural interventions, and this management represents another barrier to delivering adequate nutrition. Historically patients had their feeding discontinued the night before the procedure or at least 8 hours prior; current guidelines advise against this prolonged fasting period, and recommend that the time period a patient is made NPO prior to, during, and immediately following the time of diagnostic tests or procedures should be minimized to prevent inadequate delivery of nutrients and prolonged periods of ileus. lleus may be propagated by NPO status (see Table 1).

A recent retrospective observational study reported that trauma ICU patients, whose average ICU LOS was about 19 days, had EN stopped for planned procedures for a mean duration of 30 hours per patient. ${ }^{15}$ This is a significant amount of time to be without nutrients for these critically ill patients whose metabolic demands are already increased. One study found that enteral feeding was more likely to be held by experienced surgeons, anesthesiologists, and nurses. ${ }^{14}$ More specifically the study reported a strong correlation with seniority and the non-compliance of recommendations in the ICU population.

While this non-compliance is a serious concern that needs proper attention at every institution, several studies have demonstrated the influential role of NT education programs, even among the senior healthcare providers, with a rapid shift from non-compliance to compliance with basic education programs. ${ }^{8,10,11}$

\section{A TEAM APPROACH}

A practical solution to these complicated management issues is to implement a multidisciplinary team with dietitians and nutritional therapists. This team approach can improve patient outcomes and reduce overall costs. ${ }^{10,13,24}$ A study at Albert Einstein Medical Center reports that the use of NT specialists could reduce preventable costs by as much as $\$ 156,654$ per year. ${ }^{24}$ Likewise, the presence of educated NT team correlated with patients receiving more EN (6.7 vs. 5.4 per 10 patient-days) and reduced overall LOS ( 25 v 35 days). ${ }^{15}$ Without this multidisciplinary approach, the providers' lack of comfort with NT and incomplete documentation yields subpar results and, ultimately, will lead to poor patient outcomes.

\section{Conclusions}

Multiple studies have shown that NT, when properly instituted, can significantly impact patient management. Indeed, the effects of its implementation have been shown to reduce infection rates, improve wound healing, decrease both hospital and intensive care center length-of-stay, and decrease mortality. ${ }^{8,10,12,25,26}$ Studies have also demonstrated that effective application of NT can also reduce overall costs in the management of patients.

As the role of NT in medicine increases and 
the application becomes more precise, it is necessary to address the discordance between the evidence-based recommendations and usual practice amongst healthcare professionals. Providers need to be confident in knowing when and how to manage NT. As the cited studies have demonstrated, successful implementation of an NT education program improves efficiency and provider comfort with medical management. Additional resources ought to be committed to revise and optimize nutrition education programs for medical training institutions. This is a proven method that rapidly impacts the application of this knowledge and overall compliance with national guidelines.

Author Affiliations: Chase C. Hansen is a medical student at Texas Tech University Health Sciences Center in Lubbock. Sharmila Dissanaike is a faculty physician in the Department of Surgery at Texas Tech University Health Sciences Center.

Submitted: $2 / 24 / 2015$

Accepted: $3 / 21 / 2015$

Reviewers: C. Scott O'Banion PharmD

Published electronically: 4/15/2015

Conflict of Interest Disclosures: None

\section{REFERENCES}

1. Harkness L. The history of enteral nutrition therapy: from raw eggs and nasal tubes to purified amino acids and early postoperative jejunal delivery. J Am Diet Assoc 2002; 102(3):399-404.

2. Adams S, Dellinger EP, Wertz MJ, Oreskovich MR, Simonowitz D, Johansen K. Enteral versus parenteral nutritional support following laparotomy for trauma: a randomized prospective trial. J Trauma 1986; 26(10):882-891.

3. McClave SA, Martindale RG, Vanek VW, et al. Guidelines for the Provision and Assessment of Nutrition Support Therapy in the Adult Critically Ill Patient: Society of Critical Care Medicine (SCCM) and American Society for Parenteral and Enteral Nutrition (A.S.P.E.N.). JPEN J Parenter Enteral Nutr 2009 May-Jun; 33(3):277-316.

4. Kreymann KG, Berger MM, Deutz NE, et al. ESPEN Guidelines on Enteral Nutrition: Intensive care. Clin Nutr 2006 Apr; 25(2):210-23.
5. Heyland DK, Dhaliwal R, Drover JW, Gramlich L, Dodek P. Canadian clinical practice guidelines for nutrition support in mechanically ventilated, critically ill adult patients. JPEN J Parenter Enteral Nutr 2003; 27(5):355-373.

6. Harvey SE, Parrott F, Harrison DA, et al. Trial of the Route of Early Nutritional Support in Critically Ill Patients. N Engl J Med 2014; 371:1673-84.

7. Singer P, Berger MM, Van den Berghe G, et al. ESPEN guidelines on Parenteral Nutrition: intensive care. Clin Nutr 2009; 28(4):387-400.

8. Behara AS, Peterson SJ, Chen Y, Butsch J, Lateef O, Komanduri S. Nutrition support in the critically ill: a physician survey. JPEN J Parenter Enteral Nutr 2008 Mar-Apr; 32(2):1139.

9. McWhirter JP, Pennington CR. Incidence and recognition of malnutrition in hospital. BMJ 1994; 308:945-948.

10. Castro MG, Pompilio CE, Horie LM, Verotti CC, Waitzberg DL. Education program on medical nutrition and length of stay of critically ill patients. Clin Nutr 2013 Dec; 32(6):1061-6.

11. Spear S, Sim V, Moore FA, Todd SR. Just say no to intensive care unit starvation: a nutrition education program for surgery residents. Nutr Clin Pract 2013 Jun; 28(3):387-91.

12. Windle EM. Adequacy of dietetic service provision to adult critical care: a survey of 33 centres in Northern England. J Hum Nutr Diet 2007 Apr; 20(2):111-9; quiz 119-20.

13. Taylor B, Renfro A, Mehringer L. The role of the dietitian in the intensive care unit. Curr Opin Clin Nutr Metab Care 2005 Mar; 8(2):211-6.

14. Bozzetti F, Mariani L, Laviano A. Compliance of health professionals with the recommendations of the ESPEN Clinical Practice Guidelines: Results of ad hoc questionnaire. Clin Nutr ESPEN 2014 Feb; 9(1):e34-e38.

15. Ridley E, Gantner D, Pellegrino V. Nutrition therapy in critically ill patients- a review of current evidence for clinicians. Clin Nutr 2015.

16. Allison SP. Integrated nutrition. Proc Nutr Soc 2005 Aug; 64(3):319-23.

17. Darawad MW, Hammad S, Al-Hussami M, Haourani E, Aboshaiqah AE, Hamdan-Mansour AM. Investigating critical care nurses' perception regarding enteral nutrition. Nurse Educ Today 2015; 35(2):414-419.

18. Chuntrasakul, CSithamr, SChinswangwatankul, et al. Early nutritional support in severe traumatic patients. J Med Assoc Thai 1996; 79: 21-26.

19. Raguso CA, Dupertuis YM, Pichard C. The role of visceral proteins in the nutritional assessment of intensive care unit patients. Curr Opin Clin Nutr Metab Care 2003; 6(2):211-216.

20. Zaloga GP. The myth of the gastric residual volume. Crit Care Med 2005; 33:449-50. 
21. Moreira TV, McQuiggan M. Methods for the assessment of gastric emptying in critically ill, enterally fed adults. Nutr Clin Pract 2009; 24(2):261-273.

22. Reignier J, Mercier E, Le Gouge A, Boulain T. Effect of not monitoring residual gastric volume on risk of ventilator-assisted pneumonia in adults receiving mechanical ventilation and early enteral feeding. JAMA 2013; 309(3):249-56.

23. Spain DA, McClave SA, Sexton LK, et al. Infusion protocol improves delivery of enteral tube feeding in the critical care unit. JPEN J Parenter Enteral Nutr 1999; 23(5):288-292.

24. Goldstein M, Braitman LE, Levine GM. The medical and financial costs associated with termination of a nutrition support nurse. J Parenter Enteral Nutr 2000 Nov-Dec; 24(6):323-7.

25. Kudsk KA, Croce MA, Fabian TC, et al. Enteral versus parenteral feeding: effects on septic morbidity after blunt and penetrating abdominal trauma. Ann Surg 1992; 215(5):503-511; discussion 511-503.

26. Schroeder D, Gillanders L, Mahr K, Hill GL. Effects of immediate postoperative enteral nutrition on body composition, muscle function, and wound healing. JPEN J Parenter Enteral Nutr 1991; 15(4):376-383. 